\title{
Rolling Element Bearing Performance Degradation Assessment Using Variational Mode Decomposition and Gath-Geva Clustering Time Series Segmentation
}

\author{
Yaolong Li, ${ }^{1}$ Hongru Li, ${ }^{1}$ Bing Wang, ${ }^{2}$ and Hongqiang Gu${ }^{1}$ \\ ${ }^{1}$ Shijiazhuang Mechanical Engineering College, Shijiazhuang 050003, China \\ ${ }^{2}$ Shanghai Maritime University, Shanghai 200135, China \\ Correspondence should be addressed to Hongru Li; lihr168@sohu.com
}

Received 28 April 2017; Revised 23 May 2017; Accepted 5 September 2017; Published 12 October 2017

Academic Editor: Grzegorz M. Krolczyk

Copyright ( $\odot 2017$ Yaolong Li et al. This is an open access article distributed under the Creative Commons Attribution License, which permits unrestricted use, distribution, and reproduction in any medium, provided the original work is properly cited.

By focusing on the issue of rolling element bearing (REB) performance degradation assessment (PDA), a solution based on variational mode decomposition (VMD) and Gath-Geva clustering time series segmentation (GGCTSS) has been proposed. VMD is a new decomposition method. Since it is different from the recursive decomposition method, for example, empirical mode decomposition (EMD), local mean decomposition (LMD), and local characteristic-scale decomposition (LCD), VMD needs a priori parameters. In this paper, we will propose a method to optimize the parameters in VMD, namely, the number of decomposition modes and moderate bandwidth constraint, based on genetic algorithm. Executing VMD with the acquired parameters, the BLIMFs are obtained. By taking the envelope of the BLIMFs, the sensitive BLIMFs are selected. And then we take the amplitude of the defect frequency (ADF) as a degradative feature. To get the performance degradation assessment, we are going to use the method called Gath-Geva clustering time series segmentation. Afterwards, the method is carried out by two pieces of run-to-failure data. The results indicate that the extracted feature could depict the process of degradation precisely.

\section{Introduction}

Rolling element bearings (REBs) are critical components of "rotor-bearing" system in rotating machinery. Since the cruel working condition, the REBs are vulnerable. So it is important to monitor their condition to avoid catastrophic accident in modern industry. There are many ways to monitor bearings, such as vibration [1], acoustic emission [2], oil-debris [3], and ultrasound [4]. Among them, the method based on vibration signal analysis is extensively used for a bearing.

Recently, the prediction of the residual (or remaining) useful life (RUL) is a hotspot issue. To get a better bearing fault prediction result, the so-called performance degradation assessment (PDA) is a premise. The PDA contains two important aspects. One is to extract proper features that can reflect the process of degradation. The other one is to use a method to assess the REB's performance. Feature extraction is the fundamental of PDA. The feature must reveal the real performance of the REB and be sensitive to the degradation. The types of features are usually classified into three categories, time domain features, frequency domain features, and time-frequency domain features. The time-frequency domain features are always based on timefrequency analysis, combined with the concept of spectrum, entropy, and complexity, for example, the Rényi entropy [5], the permutation entropy [6], and the general mathematical morphology particle [7]. In general, mechanical equipment undergoes a complete degradation from normal stage to failure. With the increasing of the running time and deepening of degradation, the amplitude of defect frequency (ADF) is raising therewith. So the ADF can reflect the degradation of REB directly.

To get the ADF, the first thing is to locate the defect frequency. In extraction of ADF, the common method is 
to use EMD and select appropriate intrinsic mode function (IMF) components, taking the envelope spectrum, and finally get the ADF [8]. However, EMD remains an exclusively empirical algorithm, and it lacks a solid mathematical foundation. Despite numerous attempts to improve its performance, EMD is still of low efficiency and has endpoint effect and mode mixing problems. In 2014, Dragomiretskiy and Zosso [9] proposed a method called variational mode decomposition (VMD) as an alternative to EMD, which can adaptively decompose a multicomponent signal into a number of quasiorthogonal IMFs. It has been verified that VMD outperforms EMD with regard to tone detection and separation as well as noise robustness [10]. It has a good performance and high operation efficiency. Particularly, it has solid foundation for a mathematical theory. Now the method has been applied to the abnormal ECG signal detection [11], the stock market forecasting [12], wind power prediction [13], power quality classification [14], and so on.

However, the parameters of VMD are selected by experience. Jun Zhu presented a method to optimize the parameters based on the kurtosis index through artificial fish swarm algorithm [15]. Tang and Wang used Shannon entropy as an index to optimize parameters by panicle swarm algorithm [16]. In this paper, we proposed a method to optimize the parameters based on BLIMFs themselves using genetic algorithm. As to PDA, Pan et al. [17] proposed a bearing degradation assessment method based on lifting wavelet packet decomposition and fuzzy C-means clustering. Wang et al. [18] proposed a method for PDA based on mathematical morphology fractal dimension and fuzzy C-means clustering. However, when using clustering, they only considered the distance factor, and no time parameter was recommended. The clusters should be contiguous in time. From this point of view, after extraction of $\mathrm{ADF}$ from the raw signals, the assessment of bearing performance is carried out by GGCTSS.

The remainder of this paper is organized as follows. In Section 1, the theory of VMD is briefly introduced. The optimization of the parameters is proposed in Section 2. In Section 3, by carrying out two run-to-failure data, we have extracted the ADF and used GGCTSS for PDA. Our conclusions are presented in Section 4.

\section{VMD and Its Parameters}

2.1. Variational Mode Decomposition. VMD can nonrecursively decompose a real-valued multicomponent signal into a discrete number of quasiorthogonal band-limited subsignals $u_{k}$ with specific sparsity properties in the spectral domain. Each mode is compacted around a center pulsation $\omega_{k}$. For convenience, let us call these modes obtained by VMD as band-limited IMFs (BLIMFs). The VMD technique is essentially written as a constrained variational problem in [9]

$$
\begin{aligned}
\min & \left\{\sum_{k=1}^{K}\left\|\partial_{t}\left[\left(\sigma(t)+\frac{j}{\pi t}\right) * u_{k}(t)\right] \mathrm{e}^{-j w_{k} t}\right\|_{2}^{2}\right\} \\
\text { Subject to } & \sum_{k=1}^{K} u_{k}=f,
\end{aligned}
$$

where $\left\{u_{k}\right\}=\left\{u_{1}, \ldots, u_{k}\right\}$ and $\left\{\omega_{k}\right\}=\left\{\omega_{1}, \ldots, \omega_{k}\right\}$ are the decomposed BLIMFs for the set of all $K$ modes and their estimated center frequencies, respectively. The VMD process can be briefly described as follows; for further details, refer to [9].

2.2. Parameters Involved in VMD. There are six parameters for $\mathrm{VMD}$, where $K$ represents the numbers of decomposition modes and $\alpha$ represents moderate bandwidth constraint; they are two parameters that significant influence on the decomposition results. In general, the parameter $\tau$ is set up to zero, which means that the Lagrangian multiplier is effectively shut off. The parameter init is set to be one, which suggests that center frequencies of all the modes are initialized in the uniform distribution. The parameter $D C$ is set to zero, for no DC part imposed. And the parameter tol for tolerance is set as default value $1 e-6$.

2.3. The Optimization of VMD Parameters. Figure 1 shows the impact of the parameter $\alpha$. It can be seen that the parameter $\alpha$ seems to have an inverse ratio of the decomposition modes' bandwidth. In Figure 1(a), there is no high frequency mode. It seems to be a better one with $\alpha=200$ in Figure 1(b). Because the parameter init is set to be one, the bandwidth of each mode is almost the same. To optimize the parameter $K$ and $\alpha$, A criterion must be raised. We have proposed a criterion $\eta$ in formula (2).

$$
\eta=\frac{\sum_{i}^{K}\left|l-l_{0}\right|}{f_{\max }},
$$

where $l_{0}$ is the supposed frequency band of each mode and $l$ is the actual frequency band of each mode. For example, in Figure $1(\mathrm{a}), l_{0}$ is $0-2000 \mathrm{~Hz}, 2000-4000 \mathrm{~Hz}, 4000-6000 \mathrm{~Hz}$, $6000-8000 \mathrm{~Hz}$, and $8000-10000 \mathrm{~Hz}$, and the actual frequency bands of BLIMFs are $0-1396 \mathrm{~Hz}, 1426-2549 \mathrm{~Hz}$, $2900-4014 \mathrm{~Hz}, 3686-4620 \mathrm{~Hz}$, and $3926-5376 \mathrm{~Hz}$ with the $y$-axis threshold 0.001. $\eta$ is calculated to 0.4021 , since $K$ must be set as integer, but not for parameter $\alpha$. We can find the optimized $\alpha$ using genetic algorithm of each mode. The settings of genetic algorithm are using default in MATLAB and finally get the best $K$ and $\alpha$. The minimum of $K$ is 2. EMD can be used to find the maximum value of $K$. There are 10 IMFs of the signal decomposed by EMD, so the maximum of $K$ is set up to 10 .

\section{The Experimental Verification}

3.1. Experiment Illustration. The run-to-failure REB' test data are derived from IMS center [19]. The bearing test rig hosted four test bearings on a shaft. The shaft was driven by an AC motor. The rotation speed was kept constant at $2000 \mathrm{rpm}$. A radial load of $6000 \mathrm{lbs}$ was added to the shaft and bearing by a spring mechanism. All the bearings are force oil-lubricated. Four Rexnord ZA-2115 double row bearings were installed on the shaft as shown in Figure 2. The bearings had 16 rollers in each row, a pitch diameter of 2.815 inch, roller diameter of 0.331 inch, and a tapered contact angle of $15.17^{\circ}$. A high sensitivity accelerometer was installed on each bearing's 


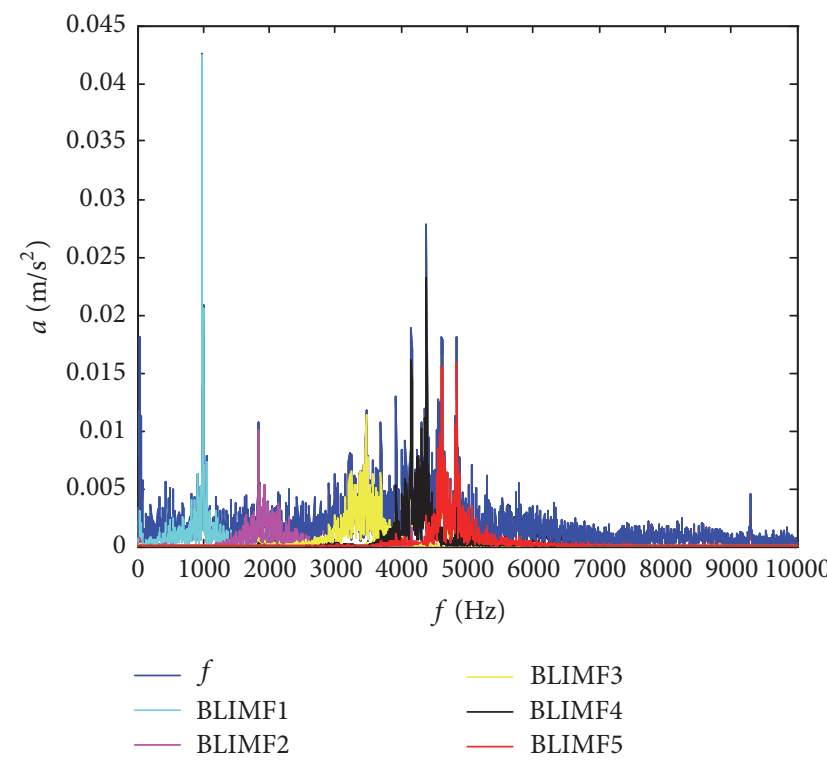

(a) $\alpha=2000$

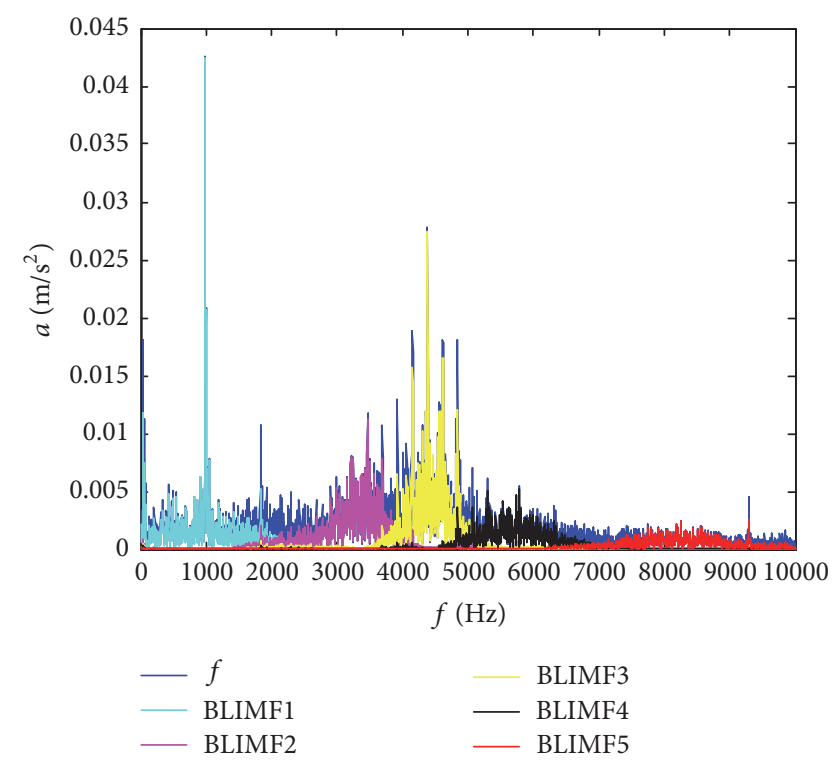

(b) $\alpha=200$

FIgURE 1: The impact of the parameter $\alpha$.
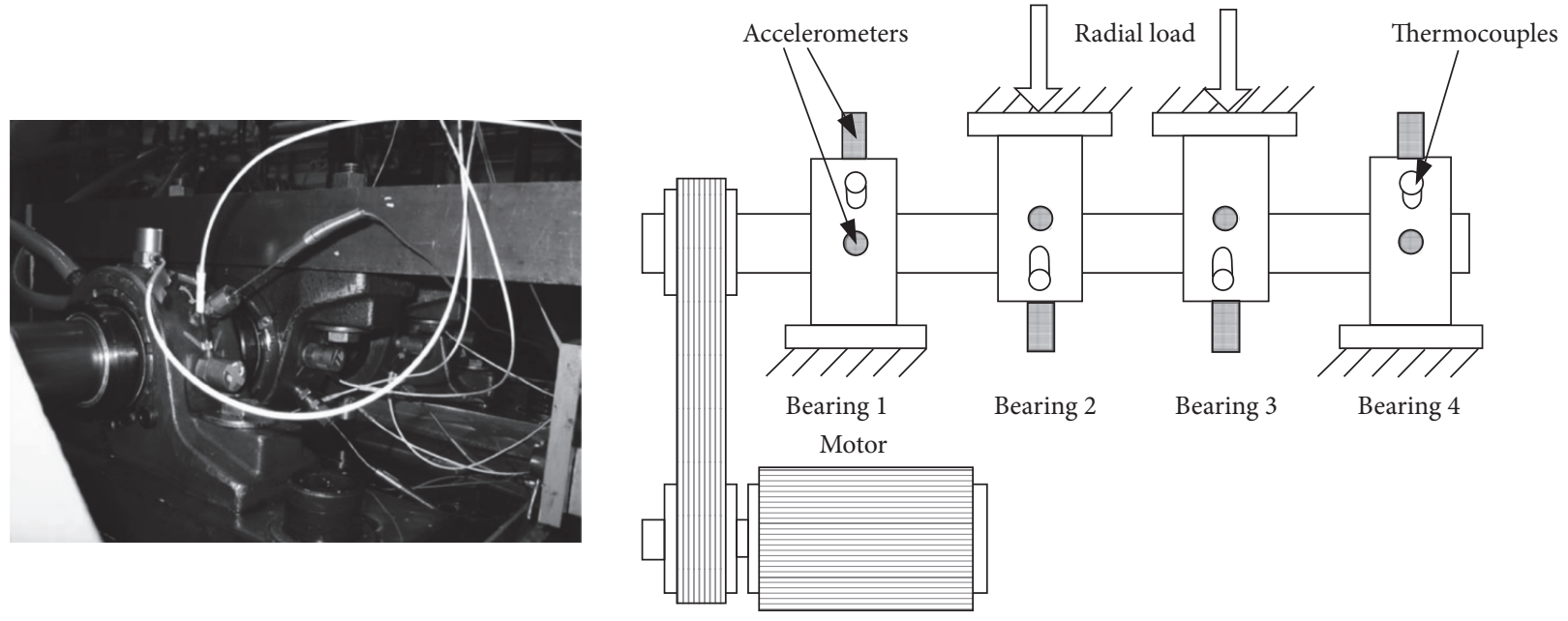

FIgURE 2: Bearing test rig.

housing. The data sampling rate was $20 \mathrm{kHz}$ and the data length was 20480 points. The sampling interval was ten minutes. We use the Bearingl_l's data of set number 2 which exhibits outer race defect as shown in Figure 3. The number of the data files is 982 (the original files number is 984 , for the last 2 files it is vague and distinctive).

For the theoretical value of outer race defect frequency, we can use the formula as follows and then have the theoretical frequency of $236.4 \mathrm{~Hz}$ :

$$
f_{o p}=\frac{Z}{2}\left(1-\frac{d}{D} \cos \theta\right) f_{r} .
$$

From Figure 4, we could not find the defect frequency of $\# 600$ (where \# means the number of files). The range of $\alpha$ is set from 1000 to 10000 . Now, by using genetic algorithm, the optimized parameter $\alpha$ of $K$ mode decomposition is presented in Table 1.

From Table 1, the optimized parameter $K$ is for 4 and $\alpha$ for 9534 . The decomposition result by optimized parameters is shown in Figure 5.

As shown in the figure, the signal is decomposed into 4 BLIMFs. The spectrum is in accordance with the order from low to high; that is, VMD has the role of band pass filtering. The BLIMFs do not show the phenomenon of mode mixing. The contrast group EMD, as shown in Figure 6, is decomposed for 11 IMFs. As we can see, EMD has a characteristic that, with the order from low to high, IMF is narrowed approximate dichotomy, and each IMF has low frequency components, and LCD and LMD have similar characteristics. At the same time, the computing time of 


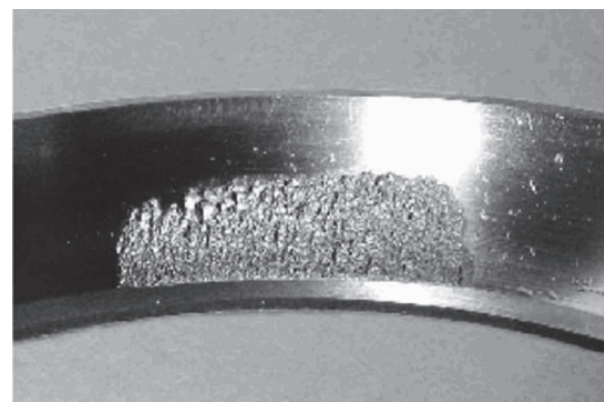

Figure 3: Outer race defect of set number 2.
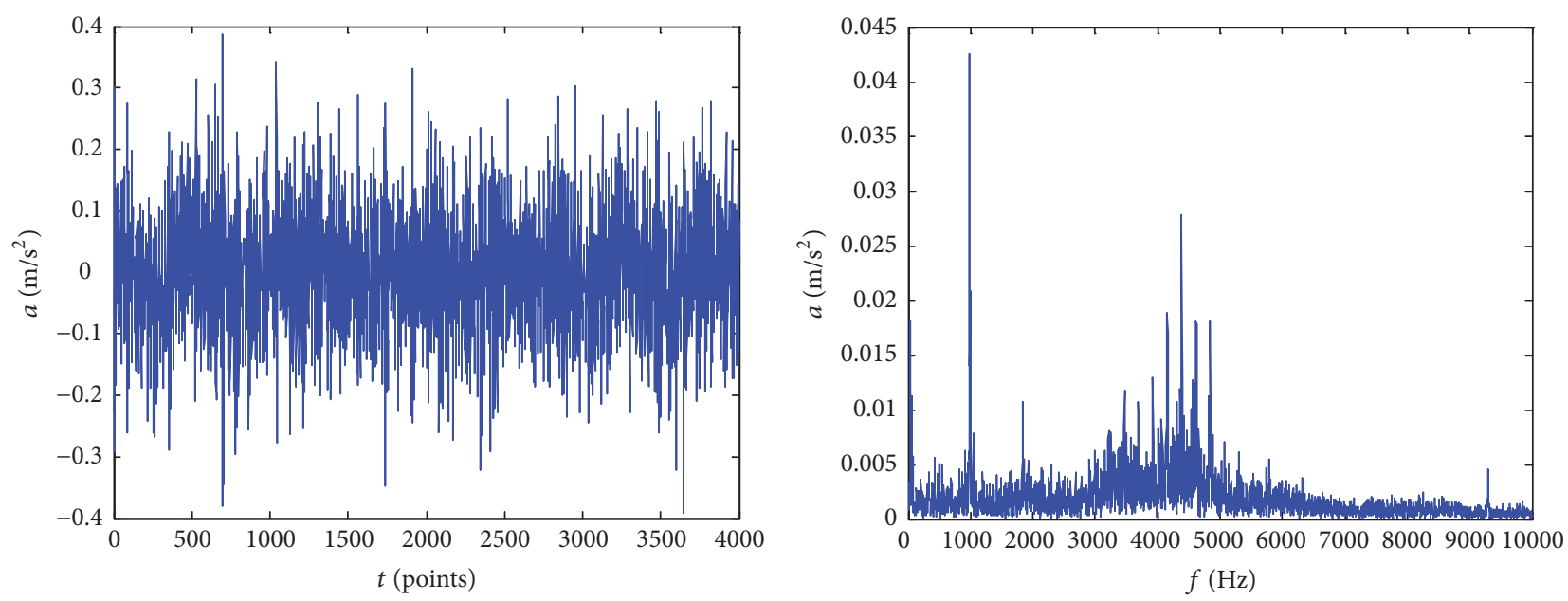

FIGURE 4: The vibration wave and frequency spectrum of $\# 600$.

TABLE 1: The optimized parameter $\alpha$ of each $K$ mode decomposition.

\begin{tabular}{lcc}
\hline$K$ & The optimized parameter $\alpha$ & Criterion $\eta$ \\
\hline 2 & 1382 & 0.9502 \\
3 & 1044 & 1.1704 \\
4 & 9534 & 0.9302 \\
5 & 9651 & 1.2129 \\
6 & 9736 & 1.5024 \\
7 & 1423 & 1.5122 \\
8 & 2321 & 1.6714 \\
9 & 1537 & 2.2026 \\
10 & 9286 & 1.5204 \\
\hline
\end{tabular}

VMD is far less than EMD, because there is no recursive iteration process of $\mathrm{VMD}$.

The signal of \#600 is decomposed into 4 BLIMFs by optimized VMD and the envelope spectrum is shown in Figure 7.

It can be seen that the BLIMF2 and BLIMF3 have the peak of $230.7 \mathrm{~Hz}$, which is close to the theoretical value 236.4 Hz. So the BLIMF2 and BLIMF3 are the right mode which have the defect frequency. By extracting the amplitude of all BLIMF2 and BLIMF3's defect frequency, an ADF of all REB's data is shown in Figure 8.

It can be seen from Figure 8 that, before \#520, the amplitude is close to zero. That indicates that there is no obvious defect before \#520. From \#520 to \#700, the ADF increases in a linear way. Guess that spalling is formed and become larger, and the REB is in the slight fault stage. About $\# 700$, there is a sudden change, and we speculate that it is caused by a bulge or a crack. From $\# 700$ to $\# 850$, the amplitude decreases and rises again. The fluctuating trend can be explained by the nature of the propagating process of the damage, when the spalling formed and later smoothed by the continuous rolling contact. As the damage spread over a broader area, the vibration level raises again. This is called "healing" phenomenon and has been stated in $[1,20$, 21]. Form $\# 850$ to the end \#982, the condition of REB is becoming fierce. The "healing" phenomenon expands, and the variances enlarge. Overall, the ADF is increasing. If the $\mathrm{ADF}$ is segmented in 4 parts, it is rational for \#1 to \#520 for normal stage, \#521 to \#699 for slight fault stage, \#700 to \#850 for serious stage, and \#851 to end for fault stage.

3.2. The PDA Using GGCTSS. As mentioned before, fuzzy $\mathrm{C}$-means clustering has its drawback for PDA. As shown in 

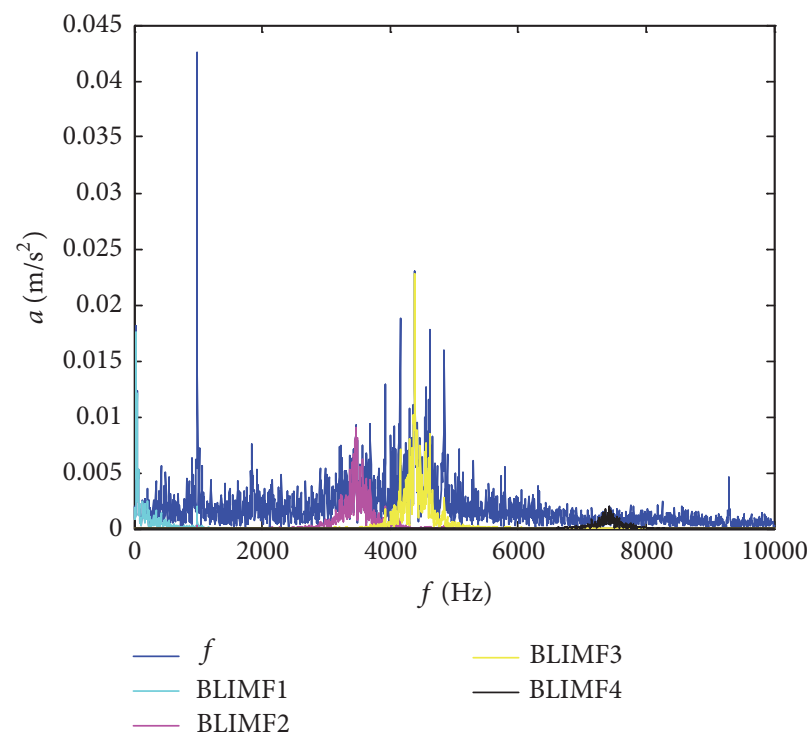

FIGURE 5: The BLIMFs and frequency spectrum of $\# 600$.
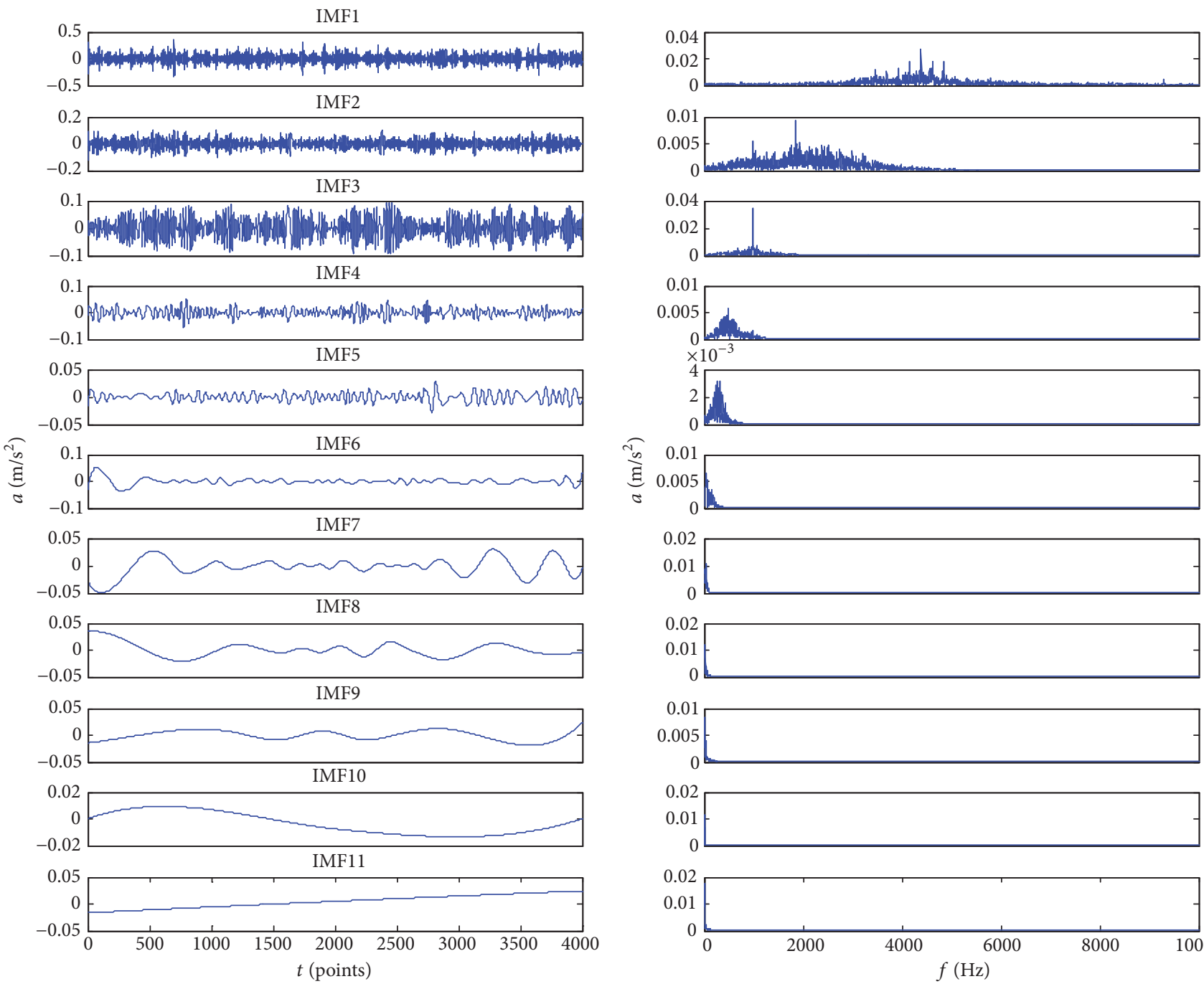
$\times 10^{-3}$

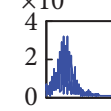
$\begin{array}{lr}\text { حैल } & 0.01 \\ \text { है } & 0.005 \\ 0 & 0\end{array}$
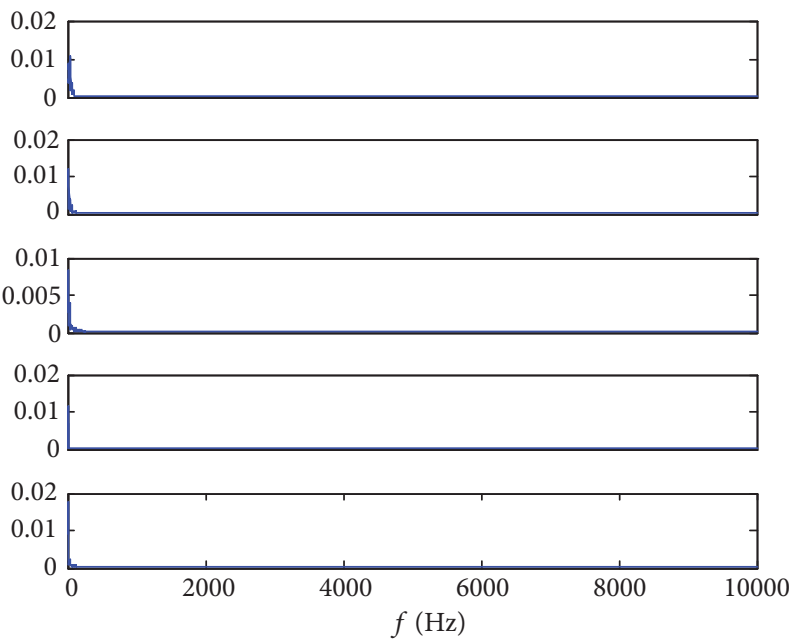

FIgURE 6: The IMFs and frequency spectrum of $\# 600$. 


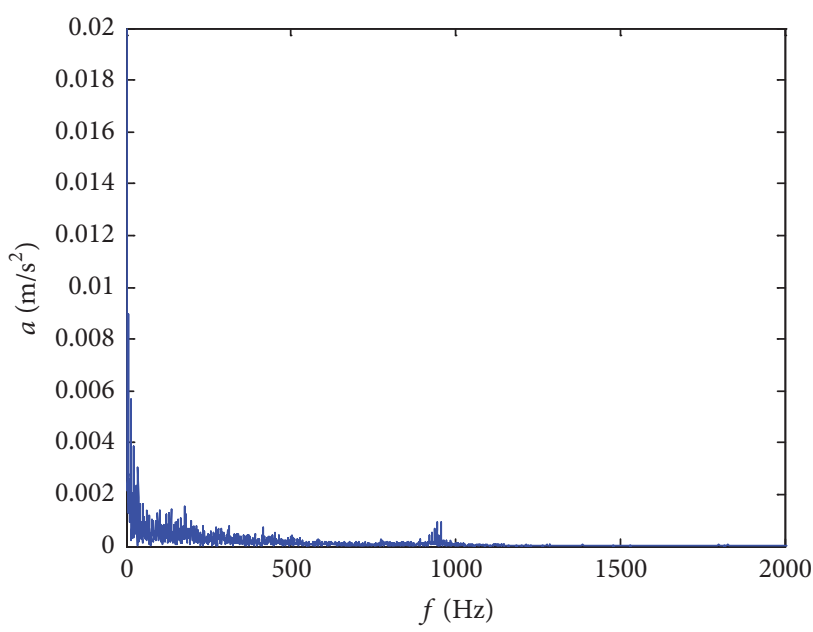

(a) BLIMF1's envelope

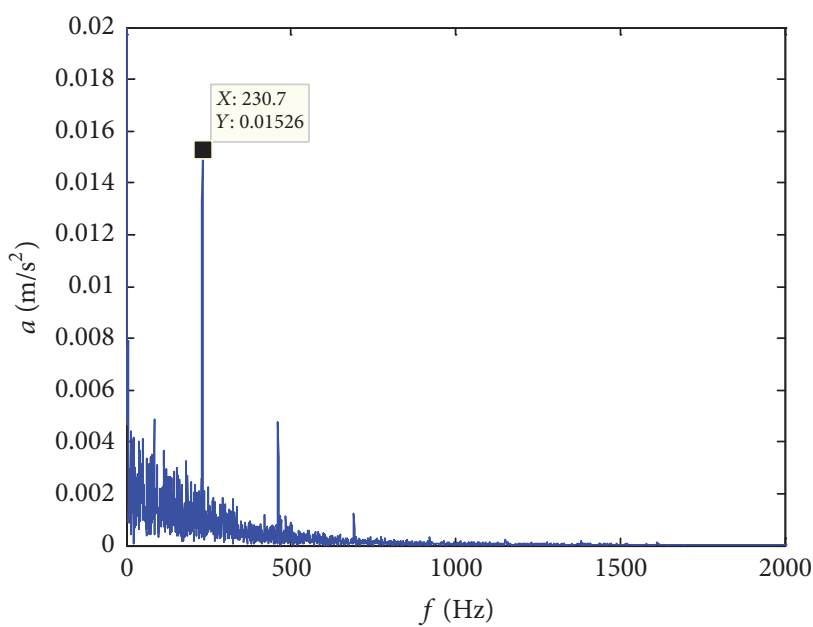

(c) BLIMF3's envelope

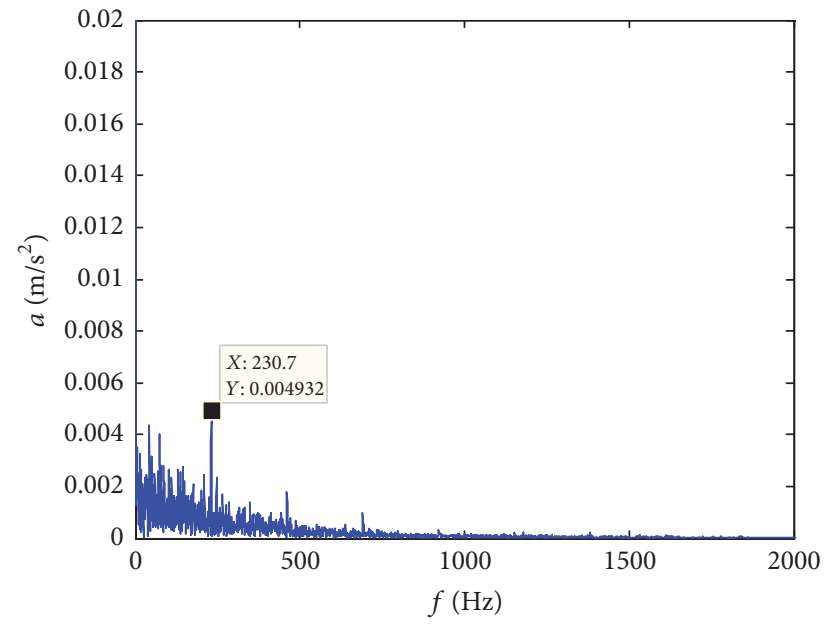

(b) BLIMF2's envelope

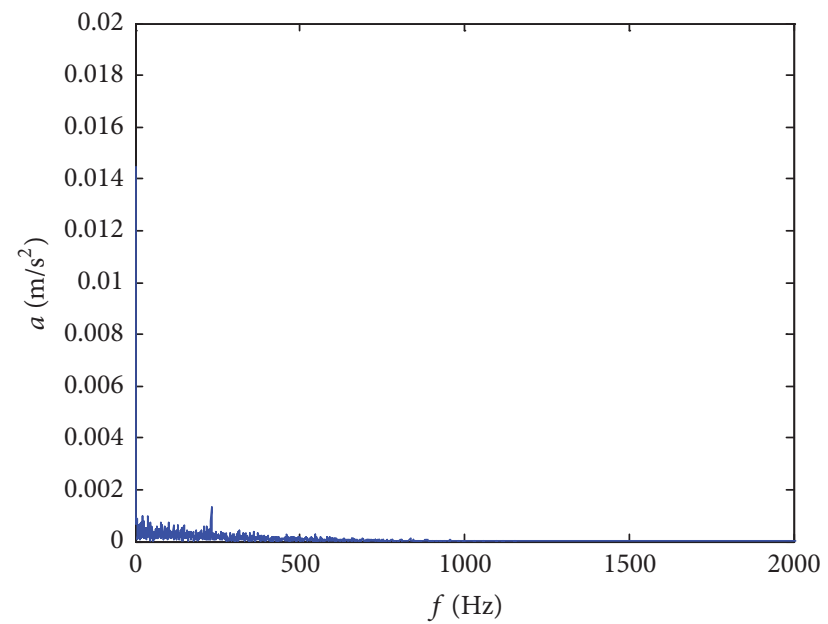

(d) BLIMF4's envelope

FIGURE 7: The envelope spectrum of BLIMF1 to BLIMF4 of \#600.

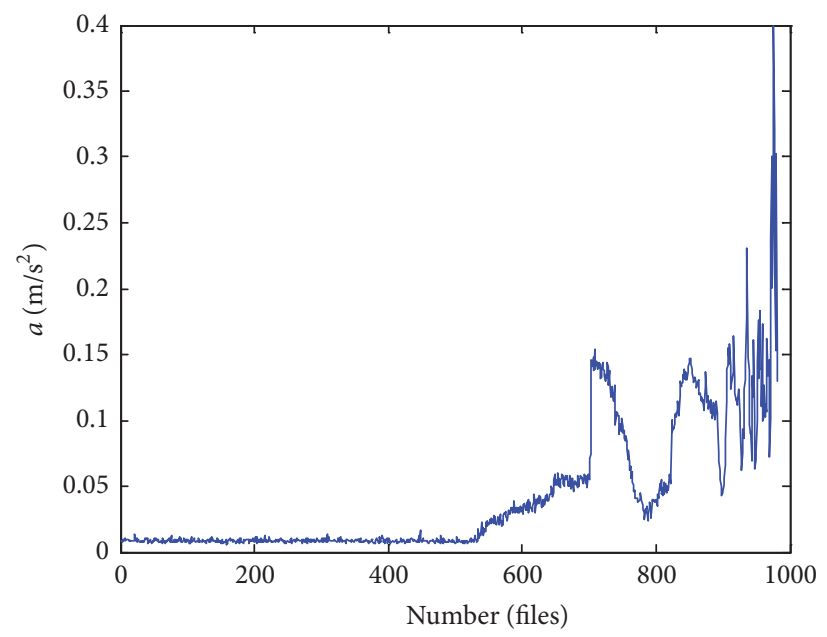

FIgure 8: The ADF of the data. 


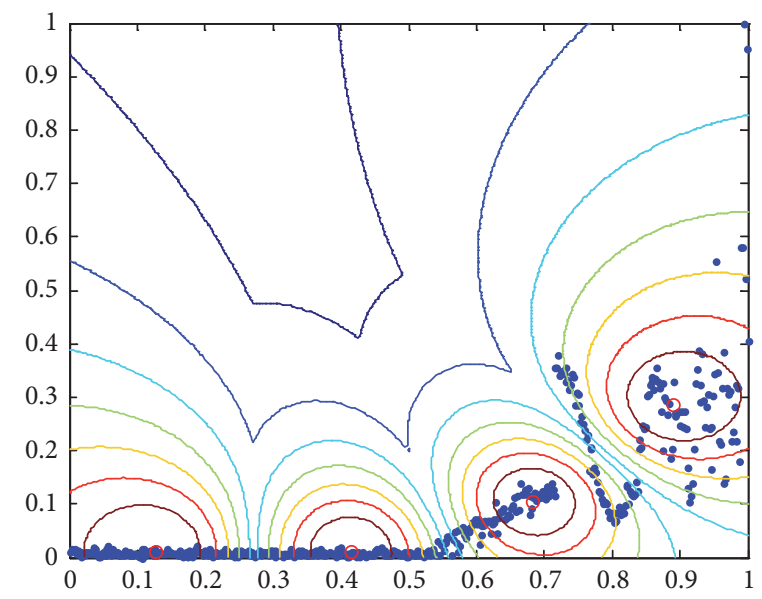

FIGURE 9: The clustering result of the ADF proposed by fuzzy C-means.

Figure 9. The four clustering results of the ADF are conducted by fuzzy C-means (the data have been normalized).

According to the clustering order from left to right, the first two clustering centers are in the normal state of the REB. The third center is in the slight fault state. The fourth one is almost close to the boundary of severely fault and failure. The result is unacceptable. References $[17,18]$ both used fuzzy C-means to realize PDA, Where owing to the degradation features which they have proposed is roughly monotonic, the cluster results of fuzzy C-means are basically distributed in time growth order. If the degradation features are inconspicuous or existing oscillation, we could not get a good clustering result. Therefore, it is necessary to import the concept of time series segmentation.

A sequence of $N$ times observed data, $T=\left\{x_{1}, x_{2}\right.$, $\left.\ldots, x_{N}\right\}$, is called time series. Time series segmentation is to find a partitioning of $T$ into $c$ segments that are internally homogeneous. After a segmentation, $T=\left\{x_{1}, x_{2}, \ldots, x_{N}\right\}$ can be partitioned into $\left\{S_{N}^{1}, S_{N}^{2}, \ldots, S_{N}^{c}\right\}$, where $S_{N}^{1}=S\left(1, t_{1}\right)$, $S_{N}^{n}=S\left(t_{n-1}+1, t_{n}\right)$, and $0<t_{1}<\cdots<t_{n-1}<\cdots<t_{c}=N$. The times $t_{1}, \ldots, t_{n-1}, \ldots, t_{c}$ are called segment boundaries or breaks, and the number $c$ is called the order of the segmentation. This segmentation is a crisp one. A time series segmentation problem can convert into an optimization. Himberg et al. [22] have defined the cost function. Under the definition, Kehagias et al. [23] proposed a dynamic programming (DP) segmentation procedure and Gedikli et al. [24] improved it with modified dynamic programming (mDP). Both methods are based on minimization of the summation of cost functions followed by distances between actual values and constant or linear fittings.

Expanding and improving the crisp bounds of the segmentation, Abonyi et al. [25] developed an algorithm for dividing time series into fuzzy segments, which considered time series segmentation as Gath-Geva clustering with time parameter as an additional variable. The GGCTSS used local probabilistic principal component analysis (PPCA) models to measure the homogeneity of the segments and fuzzy sets to represent segments in time. The function to be optimized can be written as

$$
J=\sum_{i=1}^{c} \sum_{k=1}^{N}\left(\mu_{i, k}\right)^{m} D^{2}\left(z_{k}, \eta_{i}\right),
$$

where the data point $z_{k}=\left[t_{k}, x_{k}^{T}\right]^{T}$ can be effectively modeled as a mixture of multivariate Gaussian distribution and $\mu_{i, k}$ stands for the $i$ th segment membership of $z_{k}$. The term $m$ is weighing exponent of fuzzy degree, $m \in[1, \infty]$. For general, $m=2$. The procedure of the GGCTSS can be written as follows:

(1) Initial the order segmentation $c$, iteration number $s 1$, and the threshold for compatible cluster merging ( 0.75 as default).

(2) Input the number of principal components $q$ and make sure that the sum of the anterior $q$ eigenvalues account for more than $98 \%$ accuracy of the sum of all eigenvalues.

(3) Finish the iteration and give the results of segmentation.

The results include the fuzzy segmentation of a time series, $\beta_{k}\left(t_{i}\right)$, which is defined as

$$
\beta_{k}\left(t_{i}\right)=\frac{A_{k}\left(t_{i}\right)}{\sum_{k=1}^{K} A_{k}\left(t_{i}\right)} \in[0,1],
$$

where $A_{k}\left(t_{i}\right)$ is Gaussian membership function given by

$$
A_{k}\left(t_{i}\right)=\exp \left(-\frac{1}{2} \frac{\left(t_{i}-\mu_{k}\right)^{2}}{\sigma_{k}^{2}}\right) .
$$

See [22] for more details of the method.

The $\mathrm{mDP}$ algorithm is used to treat the ADF for the fourth-order segmentations, and the results are shown in Figure 10.

It can be seen that the segmentations by $\mathrm{mDP}$ algorithm are not good. So, we use GGCTSS for PDA. The process of life was divided into 4 segments. Set the iteration to be 100 times. 


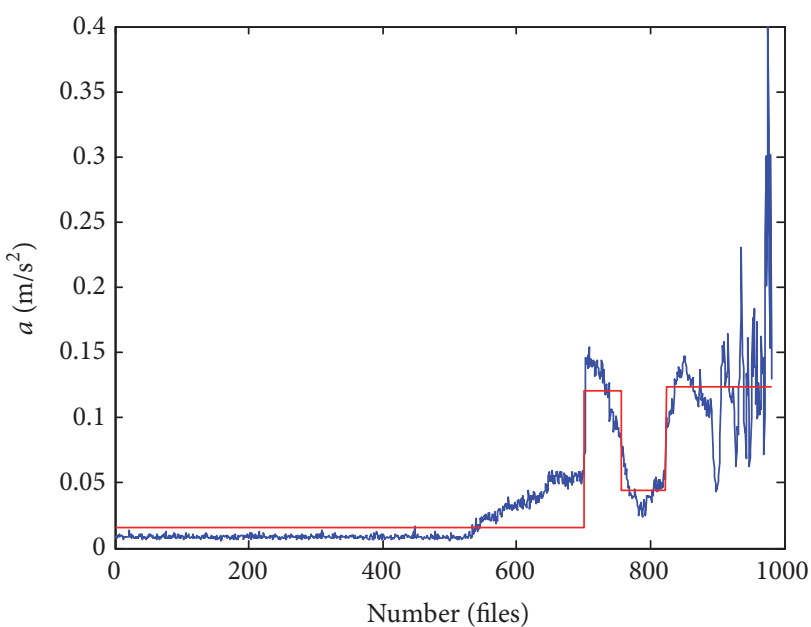

(a)

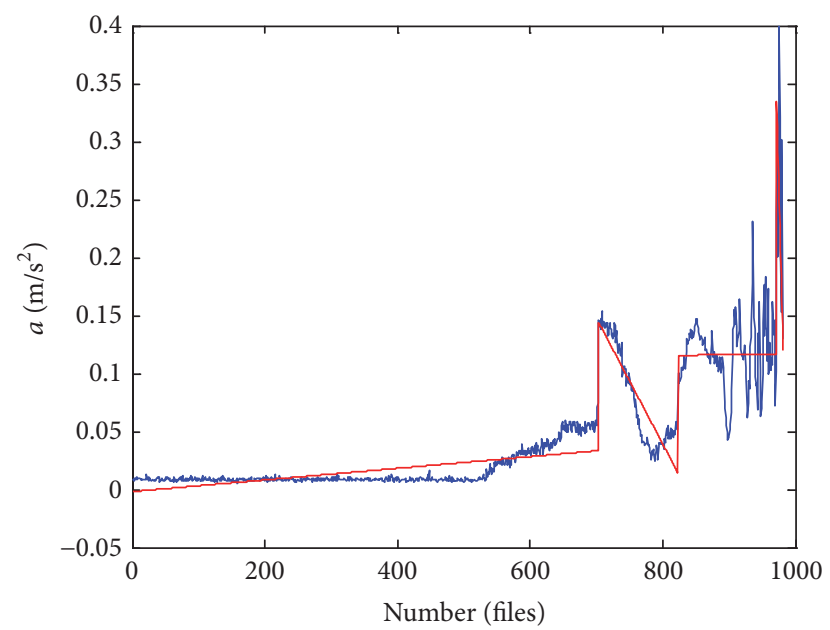

(b)

FIGURE 10: The optimal fourth-order segmentations of ADF by mDP algorithm. (a) Constant fitting and (b) linear fitting.
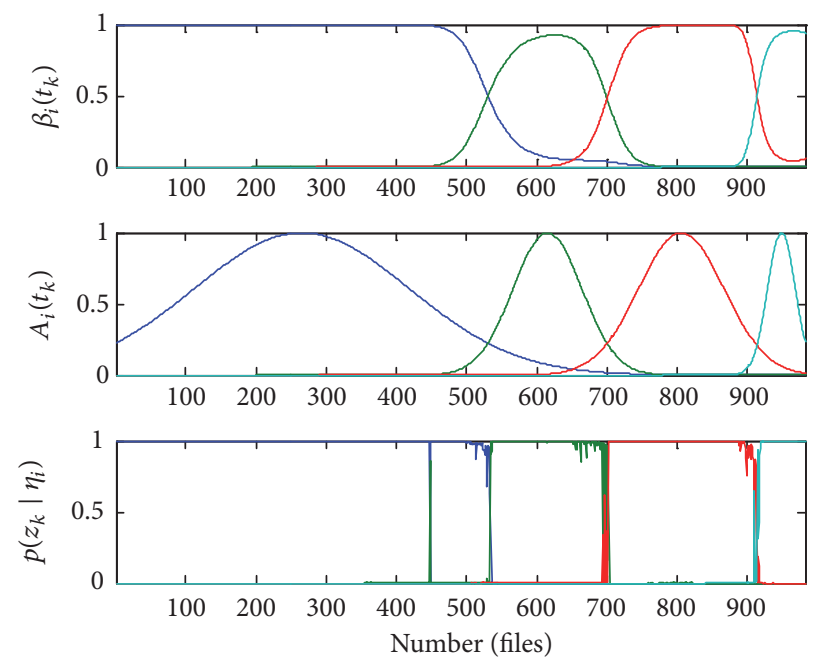

Figure 11: The result of the ADF based on GGCTSS.

It is about 70 iterations that the result is basically unchanged. Figure 11 shows the result.

It can be seen that the four segmentations are partitioned according to the order of time. The normal stage is from \#0 to $\# 522$, the slight stage is from \#522 to \#700, the severe stage is from \#700 to \#900, and the failure stage is from \#900 to \#982. It is possible to show that GGCTSS can be a good one for PDA.

3.3. Another Experiment Verification. IEEE PHM 2012 Prognostic Challenge data is another REBs' data. Figure 12 shows the experiment rig, the PRONOSTIA platform, where the data included three different loads for $1800 \mathrm{rpm}$ and $4000 \mathrm{~N}, 1650 \mathrm{rpm}$ and $4200 \mathrm{~N}$, and $1500 \mathrm{rpm}$ and $5000 \mathrm{~N}$. The sampling frequency was $25.6 \mathrm{kHz}$, and 2560 samples were recorded each 10 seconds. There were 6 pieces of runto-failure data which were the learning sets, and 11 test bearings were truncated so that participants were supposed to predict the remaining life (see Table 2) and thereby preformed RUL estimating. The learning set was quite small while the spread of the life duration of all bearings was very wide. Performing good estimates was thereby difficult and this made the challenge more exciting. More details can be seen in [26].

The data is difficult to analysis because we know nothing about the nature and the origin of the degradation: inner or outer races. We are going to use Bearingl_l's data and the characteristics of the tested bearing are shown in Table 3. And the characteristics' frequency of REB is $168.3 \mathrm{~Hz}$ for outer race, 221.7 Hz for inner race, $107.7 \mathrm{~Hz}$ for ball race, and $12.9 \mathrm{~Hz}$ for cage.

The numbers of Bearing1_1 are 2803, thus taking \#2600 for analysis, since the REB is almost run to failure. Figure 13 shows the signal.

Now, by using genetic algorithm, we have the optimized $K$ and $\alpha$, and $K$ is for 3 and $\alpha$ for 4032. The signal is 
TABLE 2: Datasets of PHM 2012 Prognostic Challenge.

\begin{tabular}{|c|c|c|c|}
\hline \multirow[b]{2}{*}{ Datasets } & \multicolumn{3}{|c|}{ Operating conditions } \\
\hline & $\begin{array}{c}\text { Conditions } 1 \\
(1800 \mathrm{rpm} \text { and } 4000 \mathrm{~N})\end{array}$ & $\begin{array}{c}\text { Conditions } 1 \\
(1650 \mathrm{rpm} \text { and } 4200 \mathrm{~N})\end{array}$ & $\begin{array}{c}\text { Conditions } 1 \\
(1500 \mathrm{rpm} \text { and } 5000 \mathrm{~N})\end{array}$ \\
\hline \multirow{2}{*}{ Learning set } & Bearing1_1 & Bearing2_1 & Bearing3_1 \\
\hline & Bearing1_2 & Bearing2_2 & Bearing3_2 \\
\hline \multirow{5}{*}{ Test set } & Bearing1_3 & Bearing2_3 & \multirow{5}{*}{ Bearing3_3 } \\
\hline & Bearing1_4 & Bearing2_4 & \\
\hline & Bearing1_5 & Bearing2 55 & \\
\hline & Bearing1_6 & Bearing2_6 & \\
\hline & Bearing1_7 & Bearing2_7 & \\
\hline
\end{tabular}

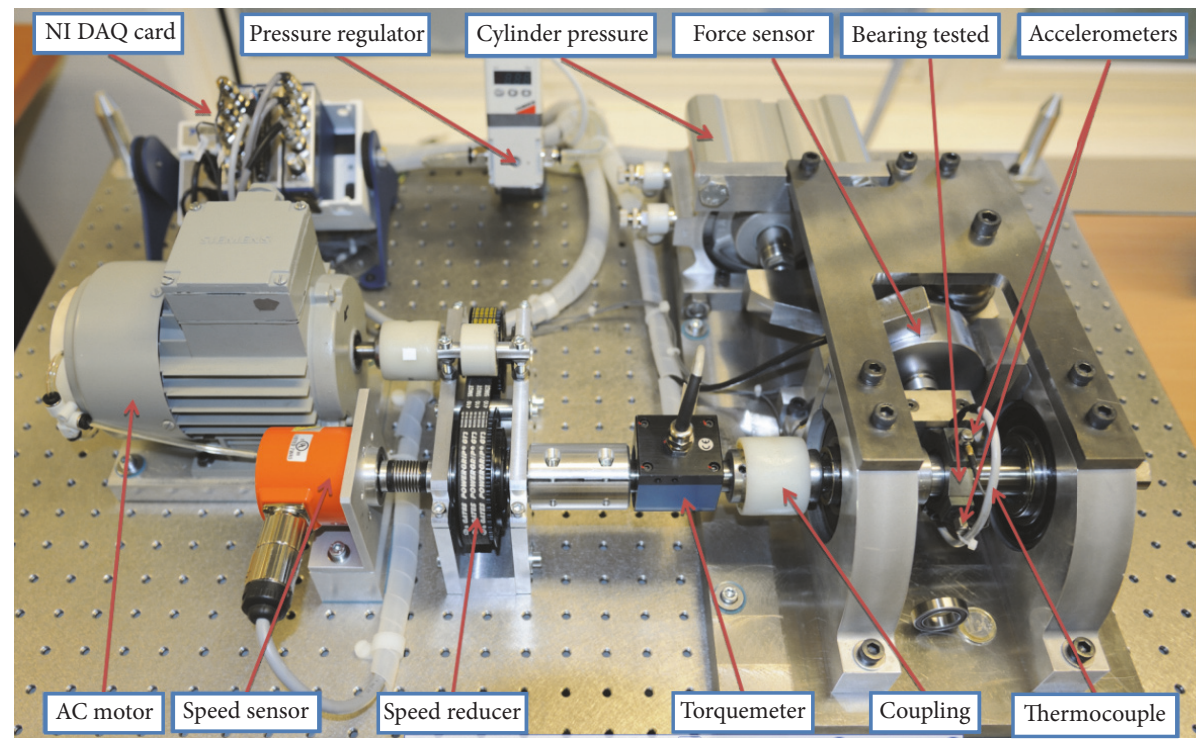

Figure 12: Overview of PRONOSTIA.

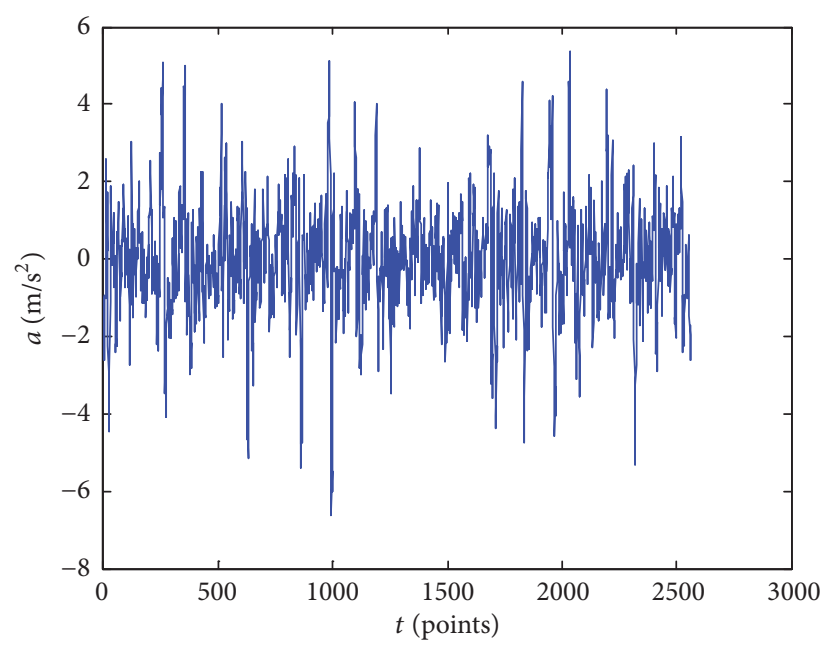

FIGURE 13: The signal of \#2600. 


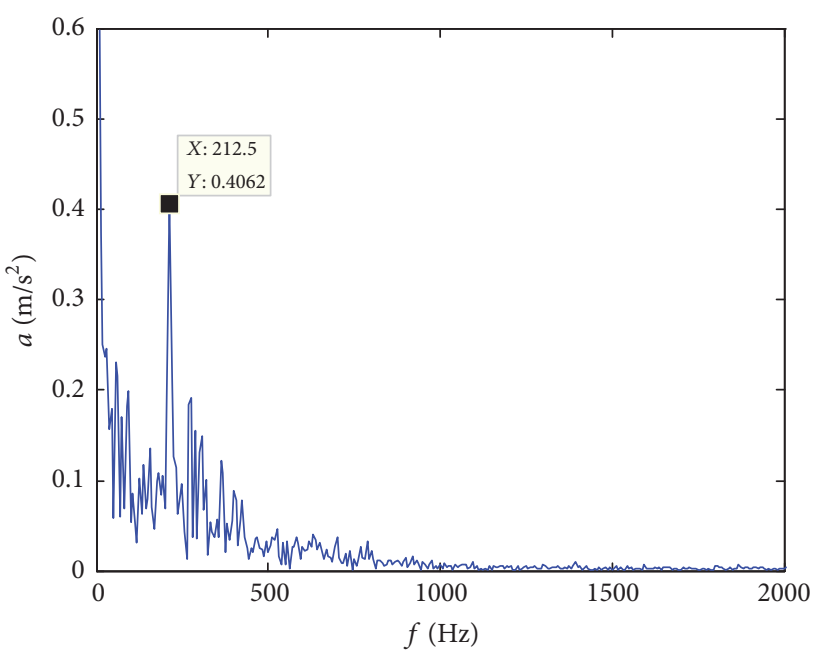

(a) BLIMF1's envelope

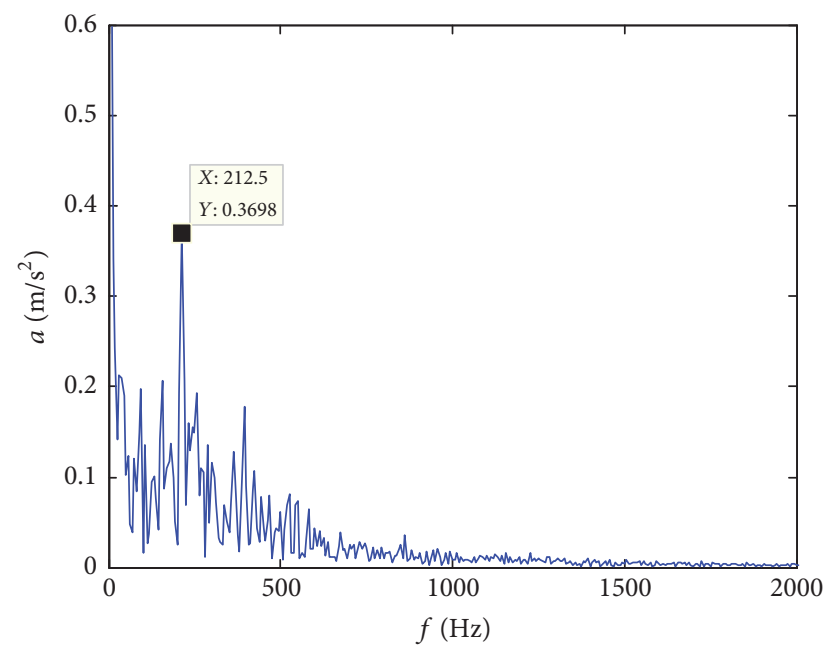

(b) BLIMF2's envelope

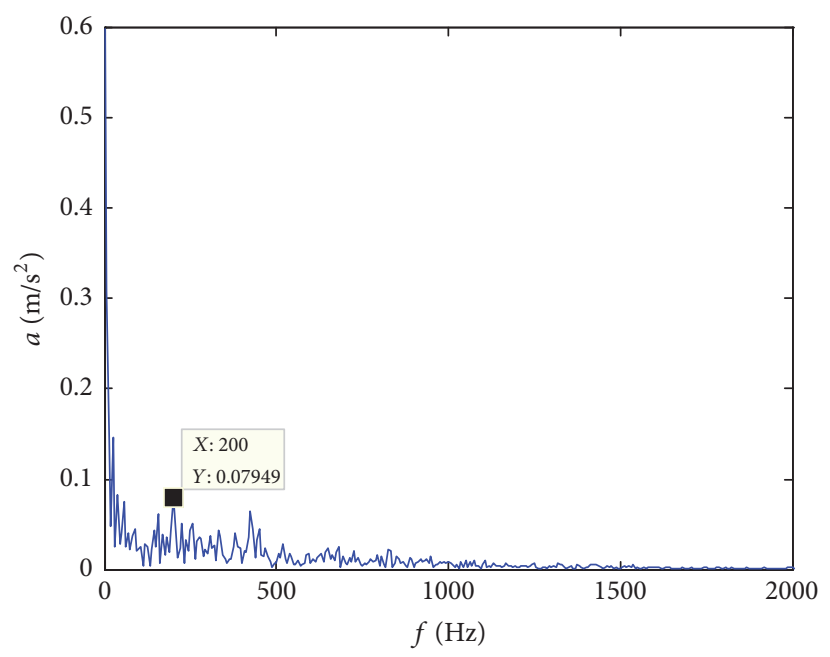

(c) BLIMF3's envelope

FIGURE 14: The envelope spectrum of BLIMF1 to BLIMF3 of \#2600.

TABLE 3: Characteristics of tested bearings.

\begin{tabular}{lc}
\hline$Z$ (ball numbers of the REB) & 13 \\
$d$ (diameter of the REB) & $3.5 \mathrm{~mm}$ \\
$D m$ (bearing mean diameter) & $25.6 \mathrm{~mm}$ \\
$r$ (rotation rate) & $1800 \mathrm{r} / \mathrm{min}$ \\
\hline
\end{tabular}

decomposed into 3 BLIMFs and the envelope spectrum is shown in Figure 14. It is obvious that BLIMF1 and BLIMF2 have the frequency of inner defect. So Bearing1_1 gets inner defect finally.

By extracting the amplitude of all BLIMF1 and BLIMF2' defect frequency, an ADF of all Bearing1_l's data is shown in Figure 15.

From Figure 15, there are no evident boundaries of each degradation stage. At the end of the bearing's life, there is a sudden increase. If we set the segment number as 3 , the result is shown in Figure 16. The normal stage is from \#0 to \#1432, slight stage is from \#1433 to \#2264, and severe stage is from \#2265 to \#2803.

\section{Conclusions}

This paper has proposed a method to assess the performance degradation of REB using VMD and GGCTSS. We have given a novel method to optimize the VMD parameter. By using GGCTSS, we can effectively segment the degradation process. From the above analysis, we can conclude that

(1) the ADF extracted by VMD can commendably reflect the degradation development of REBs;

(2) compared with crisp time series segmentation, the GGCTSS is more suitable for PDA of REBs.

However, there are also some problems that should be focused on in the future study, for example, to optimize the selection of GGCTSS segment number. 


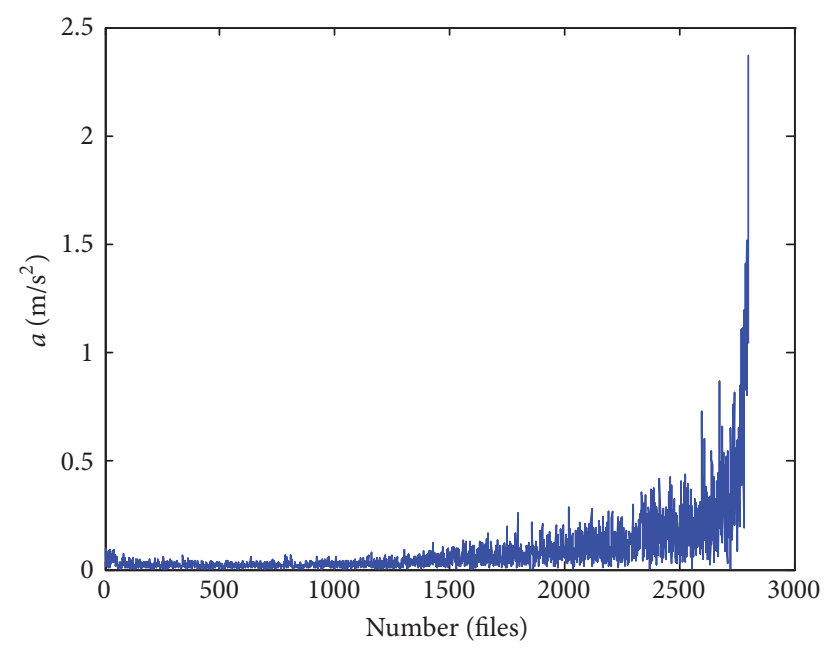

Figure 15: The ADF of the Bearing1_1.
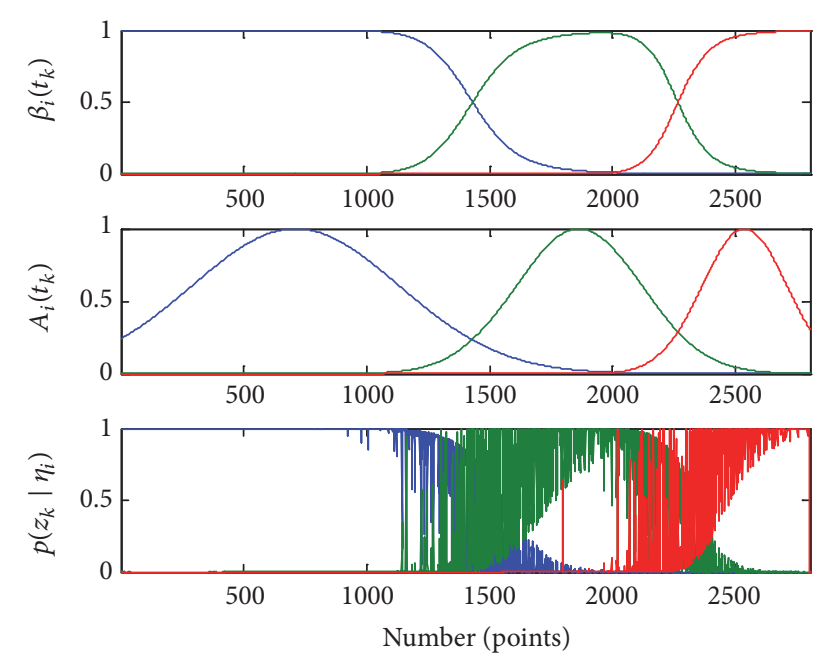

Figure 16: The result of Bearing1_l's ADF based on GGCTSS.

\section{Conflicts of Interest}

The authors declare that they have no conflicts of interest.

\section{Acknowledgments}

This project is supported by National Natural Science Foundation of China (Grant no. 51541506). The authors also appreciate the Centre for Intelligent Maintenance System and FEMTO-ST Institute for providing the experimental data.

\section{References}

[1] I. El-Thalji and E. Jantunen, "A summary of fault modelling and predictive health monitoring of rolling element bearings," Mechanical Systems and Signal Processing, vol. 60-61, pp. 252272, 2015.

[2] W. Caesarendra, B. Kosasih, A. K. Tieu, H. Zhu, C. A. S. Moodie, and Q. Zhu, "Acoustic emission-based condition monitoring methods: review and application for low speed slew bearing,"
Mechanical Systems and Signal Processing, vol. 72-73, pp. 134159, 2016

[3] Y. Iwai, T. Honda, T. Miyajima, S. Yoshinaga, M. Higashi, and Y. Fuwa, "Quantitative estimation of wear amounts by real time measurement of wear debris in lubricating oil," Tribology International, vol. 43, no. 1-2, pp. 388-394, 2010.

[4] S. Taylor and K. Wiggins, "Diagnosis of bearing defects using a heterodyning ultrasound detector," Journal of Failure Analysis and Prevention, vol. 15, no. 4, pp. 470-473, 2015.

[5] P. Boškoski, M. Gašperin, D. Petelin, and Đ. Juričić, "Bearing fault prognostics using Rényi entropy based features and Gaussian process models," Mechanical Systems and Signal Processing, vol. 52-53, pp. 327-337, 2015.

[6] R. Q. Yan, Y. B. Liu, and R. X. Gao, "Permutation entropy: a nonlinear statistical measure for status characterization of rotary machines," Mechanical Systems and Signal Processing, vol. 29, no. 5, pp. 474-484, 2012.

[7] H. Li, Y. Wang, B. Wang, J. Sun, and Y. Li, "The application of a general mathematical morphological particle as a novel indicator for the performance degradation assessment of a bearing," Mechanical Systems and Signal Processing, vol. 82, no. 1, pp. 490-502, 2014.

[8] M. Pan and W. Tsao, "Using appropriate IMFs for envelope analysis in multiple fault diagnosis of ball bearings," International Journal of Mechanical Sciences, vol. 69, pp. 114-124, 2013.

[9] K. Dragomiretskiy and D. Zosso, "Variational mode decomposition," IEEE Transactions on Signal Processing, vol. 62, no. 3, pp. 531-544, 2014.

[10] Y. Wang and R. Markert, "Filter bank property of variational mode decomposition and its applications," Signal Processing, vol. 120, pp. 509-521, 2016.

[11] R. K. Tripathy, L. N. Sharma, and S. Dandapat, "Detection of shockable ventricular arrhythmia using variational mode decomposition," Journal of Medical Systems, vol. 40, no. 4, article no. 79, pp. 1-13, 2016.

[12] S. Lahmiri, "Intraday stock price forecasting based on variational mode decomposition," Journal of Computational Science, vol. 12, pp. 23-27, 2016.

[13] Y. Zhang, K. Liu, L. Qin, and X. An, "Deterministic and probabilistic interval prediction for short-term wind power generation based on variational mode decomposition and machine learning methods," Energy Conversion and Management, vol. 112, pp. 208-219, 2016.

[14] A. A. Abdoos, P. K. Mianaei, and M. R. Ghadikolaei, "Combined VMD-SVM based feature selection method for classification of power quality events," Applied Soft Computing, vol. 38, pp. 637646, 2016.

[15] J. Zhu, C. Wang, Z. Hu, F. Kong, and X. Liu, "Adaptive variational mode decomposition based on artificial fish swarm algorithm for fault diagnosis of rolling bearings," Proceedings of the Institution of Mechanical Engineers, Part C: Journal of Mechanical Engineering Science, vol. 231, no. 4, pp. 635-654, 2017.

[16] G. Tang and X. Wang, "Parameter optimized variational mode decomposition method with application to incipient fault diagnosis of rolling bearing," Journal of Xian Jiaotong University, vol. 49, no. 5, pp. 73-81, 2015.

[17] Y. Pan, J. Chen, and X. Li, "Bearing performance degradation assessment based on lifting wavelet packet decomposition and fuzzy c-means," Mechanical Systems and Signal Processing, vol. 24, no. 2, pp. 559-566, 2010. 
[18] B. Wang, H.-R. Li, Q.-H. Chen, and B.-H. Xu, "Rolling bearing performance degradative state recognition based on mathematical morphological fractal dimension and fuzzy center means," Acta Armamentarii, vol. 36, no. 10, pp. 1982-1990, 2015.

[19] H. Qiu, J. Lee, J. Lin, and G. Yu, "Wavelet filter-based weak signature detection method and its application on rolling element bearing prognostics," Journal of Sound and Vibration, vol. 289, no. 4-5, pp. 1066-1090, 2006.

[20] T. Williams, X. Ribadeneira, S. Billington, and T. Kurfess, "Rolling element bearing diagnostics in run-to-failure lifetime testing," Mechanical Systems and Signal Processing, vol. 15, no. 5, pp. 979-993, 2001.

[21] I. El-Thalji and E. Jantunen, "A descriptive model of wear evolution in rolling bearings," Engineering Failure Analysis, vol. 45, pp. 204-224, 2014.

[22] J. Himberg, K. Korpiaho, H. Mannila, J. Tikanmäki, and H. T. T. Toivonen, "Time series segmentation for context recognition in mobile devices," in Proceedings of the 1st IEEE International Conference on Data Mining (ICDM '01), pp. 203-210, December 2001.

[23] A. Kehagias, E. Nidelkou, and V. Petridis, "A dynamic programming segmentation procedure for hydrological and environmental time series," Stochastic Environmental Research and Risk Assessment (SERRA), vol. 20, no. 1-2, pp. 77-94, 2006.

[24] A. Gedikli, H. Aksoy, N. E. Unal, and A. Kehagias, "Modified dynamic programming approach for offline segmentation of long hydrometeorological time series," Stochastic Environmental Research and Risk Assessment, vol. 24, no. 5, pp. 547-557, 2010.

[25] J. Abonyi, B. Feil, S. Nemeth, and P. Arva, "Modified Gath-GEVa clustering for fuzzy segmentation of multivariate time-series," Fuzzy Sets and Systems. An International Journal in Information Science and Engineering, vol. 149, no. 1, pp. 39-56, 2005.

[26] P. Nectoux, R. Gouriveau, and K. Medjaher, "An experimental platform for bearings accelerated life test," in Proceedings of the IEEE International Conference on Prognostics and Health Management, Denver, CO, USA, 2012. 


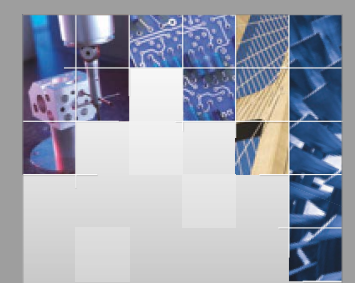

\section{Enfincering}
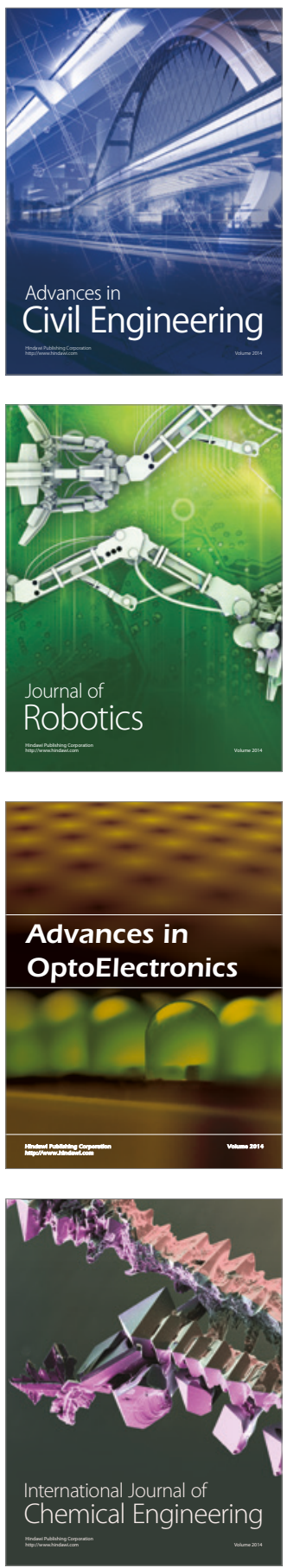

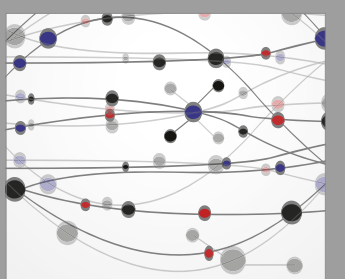

The Scientific World Journal

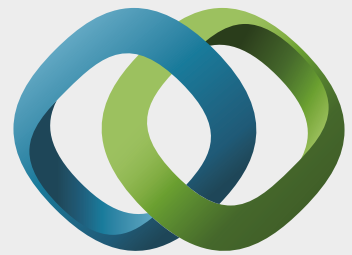

\section{Hindawi}

Submit your manuscripts at

https://www.hindawi.com
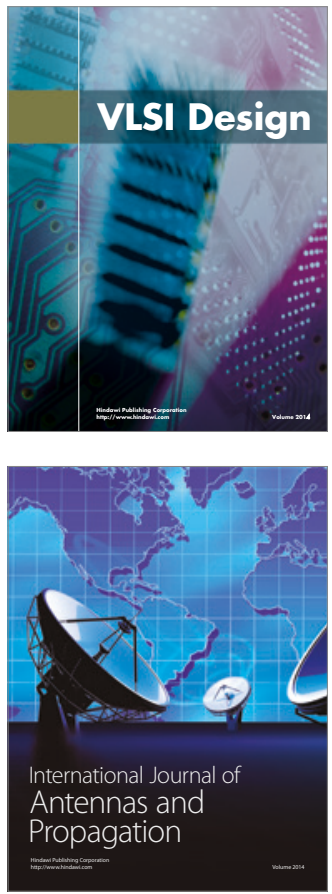

\section{Rotating}

Machinery
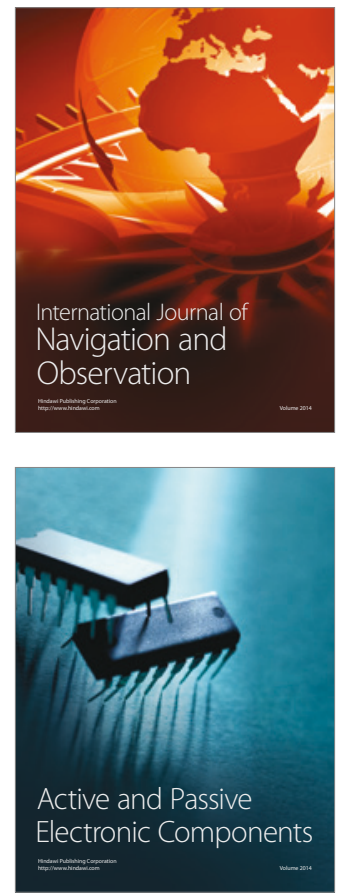
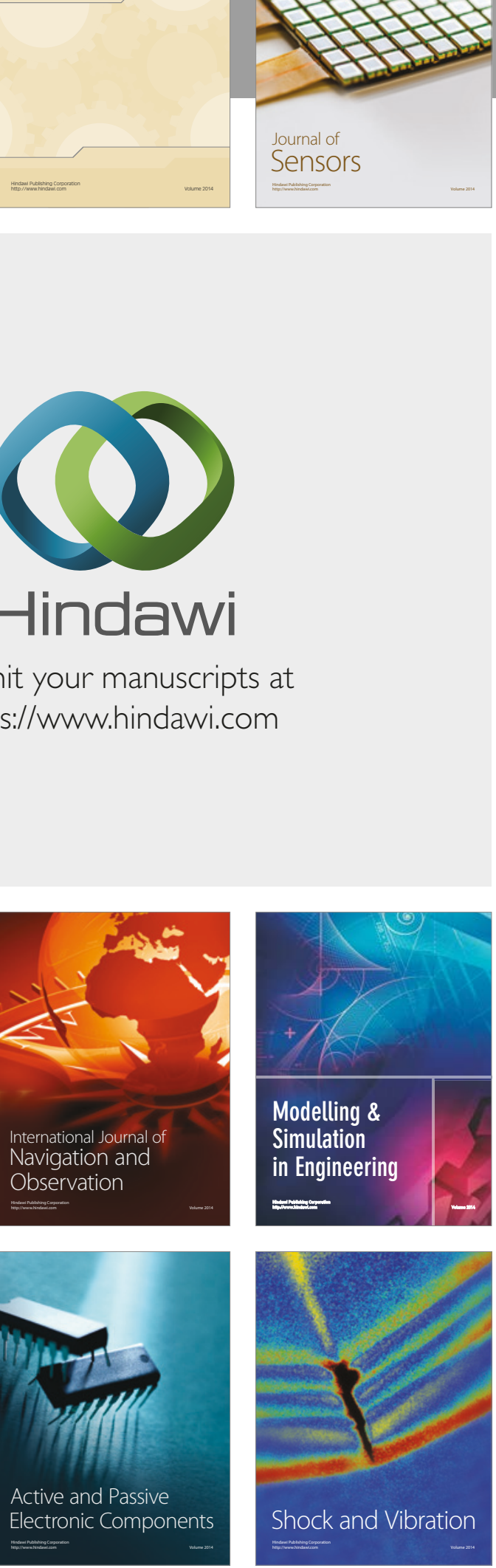
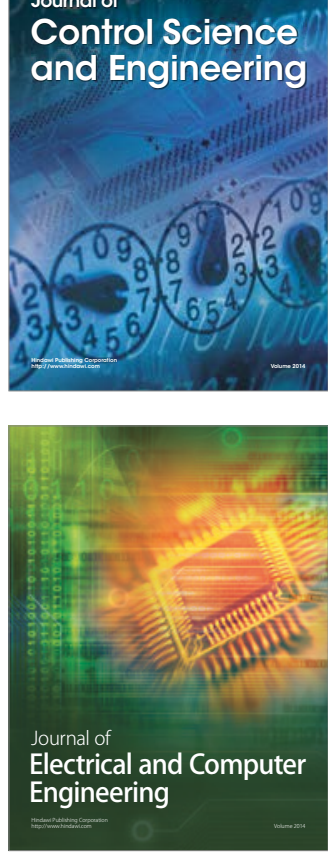

Distributed

Journal of

Control Science

and Engineering
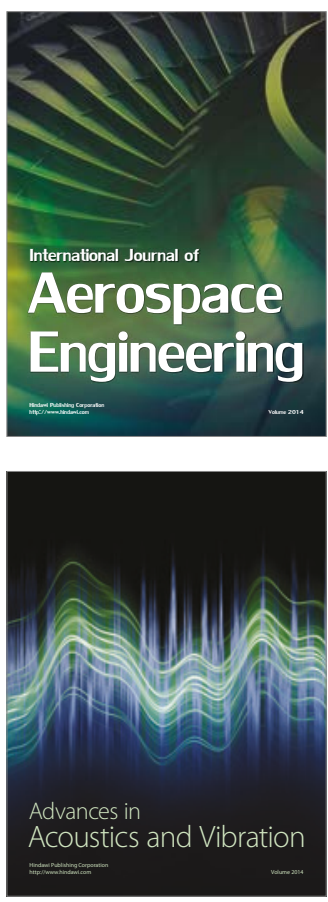

Sensor Networks 